\title{
Erratum: Fabrication and Analysis of Butterfly-type Piezoelectric Actuators
}

\author{
[J. Korean Phys. Soc. 61, 882 (2012)] \\ Won-Hee LEE and Seok-Jin YoON \\ Electronic Materials Research Center, Korea Institute of Science and Technology, Seoul 136-130, Korea \\ Chong-Yun $\mathrm{KANG}^{*}$ \\ Electronic Materials Research Center, Korea Institute of Scienece and Technology, Seoul 136-130, Korea and \\ IT-NS, KU-KIST school, Korea University, Seoul 136-701, Korea \\ Hyeong-Yup LEE \\ Piezotechnology Ind, Seoul 131-863, Korea \\ Yun-Soo LIM \\ Department of Materials Science 85 Engineering, Myongji University, Yongin 449-728, Korea \\ Byeong-Kwon Ju \\ Display and Nanosystem Laboratory, Korea University, Seoul 136-713, Korea \\ Dae-Yong JEONG \\ School of Materials Engineering, Inha University, Incheon 402-751, Korea
}

DOI: $10.3938 /$ jkps.61.1167

The acknowledgment should be corrected to 'This work was supported by the Seoul R\&D Program (JP00034) and the institutional research program of Korea Institute of Science and Technology (contract No. 2E22731)'.

*E-mail: cykang@kist.re.kr; Fax: +82-2-958-6720 